\title{
OBSERVATIONS OF THE SHELL-TYPE SUPERNOVA REMNANT CASSIOPEIA A AT TeV ENERGIES WITH VERITAS
}

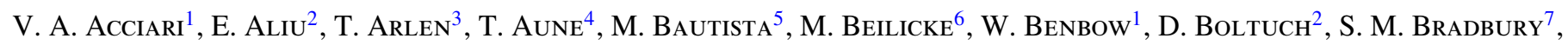

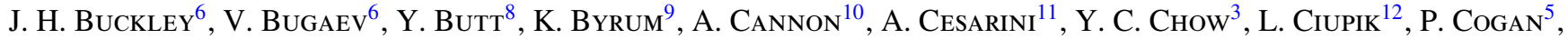
W. Cui ${ }^{13}$, R. Dickherber ${ }^{6}$, C. Duke ${ }^{14}$, T. Ergin ${ }^{8}$, S. J. Fegan ${ }^{3,24}$, J. P. Finley ${ }^{13}$, G. Finnegan ${ }^{15}$, P. Fortin ${ }^{16,24}$, L. Fortson ${ }^{12}$, A. Furniss ${ }^{4}$, N. Galante ${ }^{1}$, D. Gall ${ }^{13}$, G. H. Gillanders ${ }^{11}$, J. Grube ${ }^{10}$, R. Guenette ${ }^{5}$, G. GyuK ${ }^{12}$, D. Hanna ${ }^{5}$, J. Holder ${ }^{2}$, D. Huang ${ }^{17}$, C. M. Hui ${ }^{15}$, T. B. Humensky ${ }^{18}$, P. KaARet $^{19}$, N. Karlsson ${ }^{12}$, M. KertZman ${ }^{20}$, D. Kieda ${ }^{15}$, A. Konopelko ${ }^{17}$,*, H. Krawczynski ${ }^{6}$, F. Krennrich ${ }^{21}$, M. J. Lang ${ }^{11}$, S. LeBoheC ${ }^{15}$, G. Maier ${ }^{5,25}$, S. McArthur ${ }^{6}$, A. McCanN ${ }^{5}$, M. McCutcheon ${ }^{5}$, J. Millis ${ }^{13,26}$, P. Moriarty ${ }^{22}$, R. A. OnG ${ }^{3}$, D. PANdel ${ }^{19}$, J. S. Perkins ${ }^{1}$, M. Pohl ${ }^{21,25,27}$, J. QuinN ${ }^{10}$, K. RagaN ${ }^{5}$, P. T. Reynolds ${ }^{23}$, E. Roache ${ }^{1}$, H. J. Rose $^{7}$, M. Schroedter ${ }^{21}$, G. H. Sembroski ${ }^{13}$, A. W. Smith ${ }^{9}$, B. R. Smith ${ }^{17}$, D. Steele ${ }^{12,28}$, S. P. Swordy ${ }^{18}$, M. Theiling ${ }^{1}$, S. Thibadeau ${ }^{6}$, A. Varlotta ${ }^{13}$, V. V. Vassiliev ${ }^{3}$, S. Vincent $^{15}$,

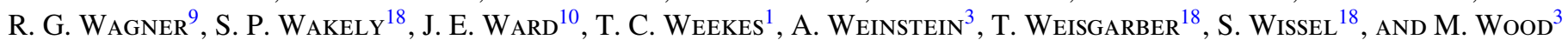

${ }^{1}$ Fred Lawrence Whipple Observatory, Harvard-Smithsonian Center for Astrophysics, Amado, AZ 85645, USA

${ }^{2}$ Department of Physics and Astronomy and the Bartol Research Institute, University of Delaware, Newark, DE 19716, USA

${ }^{3}$ Department of Physics and Astronomy, University of California, Los Angeles, CA 90095, USA

${ }^{4}$ Santa Cruz Institute for Particle Physics and Department of Physics, University of California, Santa Cruz, CA 95064, USA

${ }^{5}$ Physics Department, McGill University, Montreal, QC H3A 2T8, Canada

${ }^{6}$ Department of Physics, Washington University, St. Louis, MO 63130, USA

${ }^{7}$ School of Physics and Astronomy, University of Leeds, Leeds, LS2 9JT, UK

${ }^{8}$ Harvard-Smithsonian Center for Astrophysics, 60 Garden Street, Cambridge, MA 02138, USA

${ }^{9}$ Argonne National Laboratory, 9700 S. Cass Avenue, Argonne, IL 60439, USA

${ }^{10}$ School of Physics, University College Dublin, Belfield, Dublin 4, Republic of Ireland

${ }^{11}$ School of Physics, National University of Ireland, Galway, Republic of Ireland

12 Astronomy Department, Adler Planetarium and Astronomy Museum, Chicago, IL 60605, USA

${ }^{13}$ Department of Physics, Purdue University, West Lafayette, IN 47907, USA

${ }^{14}$ Department of Physics, Grinnell College, Grinnell, IA 50112-1690, USA

${ }^{15}$ Department of Physics and Astronomy, University of Utah, Salt Lake City, UT 84112, USA

${ }^{16}$ Department of Physics and Astronomy, Barnard College, Columbia University, NY 10027, USA

${ }^{17}$ Department of Physics, Pittsburg State University, 1701 South Broadway, Pittsburg, KS 66762, USA

${ }^{18}$ Enrico Fermi Institute, University of Chicago, Chicago, IL 60637, USA

${ }^{19}$ Department of Physics and Astronomy, University of Iowa, Van Allen Hall, Iowa City, IA 52242, USA

${ }^{20}$ Department of Physics and Astronomy, DePauw University, Greencastle, IN 46135-0037, USA

${ }^{21}$ Department of Physics and Astronomy, Iowa State University, Ames, IA 50011, USA

22 Department of Life and Physical Sciences, Galway-Mayo Institute of Technology, Dublin Road, Galway, Republic of Ireland

${ }^{23}$ Department of Applied Physics and Instrumentation, Cork Institute of Technology, Bishopstown, Cork, Republic of Ireland Received 2009 October 7; accepted 2010 February 13; published 2010 April 8

\begin{abstract}
We report on observations of very high energy $\gamma$ rays from the shell-type supernova remnant (SNR) Cassiopeia A with the Very Energetic Radiation Imaging Telescope Array System stereoscopic array of four imaging atmospheric Cherenkov telescopes in Arizona. The total exposure time for these observations is $22 \mathrm{hr}$, accumulated between September and November of 2007. The $\gamma$-ray source associated with the SNR Cassiopeia A was detected above $200 \mathrm{GeV}$ with a statistical significance of $8.3 \sigma$. The estimated integral flux for this $\gamma$-ray source is about $3 \%$ of the Crab-Nebula flux. The photon spectrum is compatible with a power law $d N / d E \propto E^{-\Gamma}$ with an index $\Gamma=2.61 \pm 0.24_{\text {stat }} \pm 0.2_{\text {sys }}$. The data are consistent with a point-like source. We provide a detailed description of the analysis results and discuss physical mechanisms that may be responsible for the observed $\gamma$-ray emission.
\end{abstract}

Key words: acceleration of particles - cosmic rays - gamma rays: ISM - ISM: individual objects (Cassiopeia A) ISM: supernova remnants

\section{INTRODUCTION}

Cassiopeia A is the youngest of the historical galactic supernova remnants (SNRs); it first appeared in the sky about 1680

\footnotetext{
${ }^{24}$ Now at Laboratoire Leprince Ringuet, Ecole Polytechnique, CNRS, IN2P3, Palaiseau, France.

${ }^{25}$ Now at DESY, Platanenallee 6, 15738 Zeuthen, Germany.

${ }^{26}$ Now at Department of Physics, Anderson University, 1100 East 5th Street, Anderson, IN 46012, USA.

${ }^{27}$ Now at Institut für Physik und Astronomie, Universität Potsdam, 14476 Potsdam-Golm, Germany.

${ }^{28}$ Now at Los Alamos National Laboratory, MS H803, Los Alamos, NM 87545, USA.

* Author to whom any correspondence should be addressed: akonopel@pittstate.edu
}

(Ashworth 1980; Stephenson \& Green 2002). The best estimate of the actual Cassiopeia A supernova (SN) explosion date is $\mathrm{AD} 1680.5 \pm 18.7$, which was deduced from the Hubble Space Telescope measurements of the expansion of ejecta knots (Fesen et al. 2006). It is also the brightest and one of the best-studied radio sources in the sky (e.g., Kassim et al. 1995). Located 3.4 kpc away (Reed et al. 1995), the optical shell of 2.5 radius corresponds to a physical size of about $2.5 \mathrm{pc}$. The synchrotron radiation of Cassiopeia A extends from radio wavelengths through the submillimeter (Mezger et al. 1986) and near-infrared (Tuffs et al. 1997) all the way to hard X-rays (Allen et al. 1997; Favata et al. 1997; Vink et al. 2001; Renaud et al. 2006). The nature of the hard X-ray emission measured up to $100 \mathrm{keV}$ still remains unclear (see Helder \& Vink 2008, albeit the morphology of the 
non-thermal X-ray emission is dominated by faint, well-defined filaments and knots (Hughes et al. 2000; Vink \& Laming 2003), which are possibly sites of cosmic ray (CR) acceleration. These energetic hadronic CRs can subsequently produce $\gamma$ rays in collisions with ambient gas via $\pi^{\circ}$-decay (Drury et al. 1994).

EGRET did not detect $\gamma$-ray emission above $100 \mathrm{MeV}$ from Cassiopeia A (Esposito et al. 1996). Likewise, first attempts to detect $\mathrm{TeV} \gamma$-ray emission from the ground with the $10 \mathrm{~m}$ Whipple telescope (Lessard et al. 1999) and with the Cherenkov Array at Themis (CAT) in the French Pyrenees (Goret et al. 1999) resulted only in upper limits. With an exposure of $232 \mathrm{hr}$, accumulated during the summer months of 1997, 1998, and 1999, HEGRA detected TeV $\gamma$-ray emission associated with Cassiopeia A (Aharonian et al. 2001). A $5 \sigma$ detection of Cassiopeia A resulted in a flux estimate of $\left(5.8 \pm 1.2_{\text {stat }} \pm 1.2_{\text {syst }}\right) \times 10^{-13} \mathrm{~cm}^{-2} \mathrm{~s}^{-1}$ above $1 \mathrm{TeV}$. The energy spectrum measured in a range from 1 to $10 \mathrm{TeV}$ was consistent with a power law with a photon index of $\Gamma=2.5 \pm 0.4_{\text {stat }} \pm 0.1_{\text {syst }}$. Recently, MAGIC and Very Energetic Radiation Imaging Telescope Array System (VERITAS) have made observations of Cassiopeia $\mathrm{A}$ at a substantially lower energy threshold.

Cassiopeia A was observed with the $17 \mathrm{~m}$ MAGIC telescope between 2006 July and 2007 January for a total exposure of $47 \mathrm{hr}$ (Albert et al. 2007). The $\gamma$-ray source was detected above $250 \mathrm{GeV}$ at the level of statistical significance of $5.2 \sigma$, with a photon flux above $1 \mathrm{TeV}$ of $\left(7.3 \pm 0.7_{\text {stat }} \pm 2.2_{\text {syst }}\right) \times$ $10^{-13} \mathrm{~cm}^{-2} \mathrm{~s}^{-1}$. The photon spectrum is compatible with a power law with an index $\Gamma=2.3 \pm 0.2_{\text {stat }} \pm 0.2_{\text {syst }}$. The source is point-like for the given angular resolution of the telescope. The position of the MAGIC source is consistent with the source position previously published by HEGRA. In addition, the energy spectrum measured by MAGIC agrees within statistical errors with that measured by HEGRA.

Here, we report on observations of Cassiopeia A with the VERITAS stereoscopic array of four imaging atmospheric Cherenkov telescopes in Arizona. In this paper, a short description of the experiment is followed by a summary of observational data and analysis results. Finally, a discussion of the physics implications of VERITAS data for existing models of TeV $\gamma$-ray emission from Cassiopeia $\mathrm{A}$ is given.

\section{EXPERIMENT}

VERITAS (Weekes et al. 2002) is an array of four imaging atmospheric Cherenkov telescopes located in southern Arizona $\left(31^{\circ} 40^{\prime} \mathrm{N}, 110^{\circ} 57^{\prime} \mathrm{W}\right)$ at an altitude of $1.3 \mathrm{~km}$. The four VERITAS telescopes are almost identical in their technical parameters (Holder et al. 2006). The $12 \mathrm{~m}$ optical reflector of a VERITAS telescope is a tessellated structure consisting of 357 identical spherical mirror facets, which are hexagonal in shape. The arrangement of the mirror facets constitutes a Davies-Cotton design (Davies \& Cotton 1957), providing a total reflecting area of $110 \mathrm{~m}^{2}$. The point-spread function (PSF) of a VERITAS telescope has an FWHM of $\sim 4^{\prime}$ on-axis (McCann et al. 2009). A high-resolution imaging camera placed at the focus of the reflector consists of 499 photomultiplier tubes (PMTs) in a close-packed hexagonal arrangement and has a field of view of 3.5. Each camera PMT views a circle of diameter 0.15 on the sky. A set of light concentrators is mounted in front of the PMTs to increase the light-collection efficiency and block the off-axis light. The camera triggers if the signal in each of any three adjacent PMTs exceeds a discriminator threshold of $50 \mathrm{mV}$, corresponding to approximately $4-5$ photoelectrons. A coincidence of at least two cameras triggering within a time gate of $100 \mathrm{~ns}$ is required to read out an event. A $48 \mathrm{~ns}$ (24 samples) length of each PMT signal is digitized with custom-built 500 Megasamples s $^{-1}$ flash analog-to-digital convertor (ADC) electronics. The nominal trigger rate of the four-telescope array was about $230 \mathrm{~Hz}$ at zenith. The cameras are flat-fielded and calibrated using nightly measured laser runs. The pedestal and pedestal variances $(\sigma)$, which provide a measure of the night sky background noise level, were calculated during each data run using pedestal events, injected at a $1 \mathrm{~Hz}$ frequency. The pedestal variances were used for computing the dynamic picture and boundary thresholds for consequent image cleaning. All PMTs with a signal exceeding the picture threshold $(5 \sigma)$ were used in the image parameterization. PMTs with a signal exceeding the boundary threshold $(2.5 \sigma)$ but lying near the picture PMTs were also selected. (e.g., see Holder et al. 2006). To characterize the shape and orientation of calibrated images recorded by each telescope, the standard second-moment parameters were calculated as described by Reynolds et al. (1993).

\section{SUMMARY OF DATA}

Cassiopeia A was observed with VERITAS for $22 \mathrm{hr}$ between September and November of 2007. All observations were made with the full four-telescope array during moonless nights. The data-analysis pipeline consists of two distinct phases. After the data are processed, the distributions from the raw data are accumulated as diagnostics of both the instrument performance and the stability of the weather conditions. Each data run is inspected for rate, timing and tracking consistency, and either accepted or rejected based on this first pass. Once this diagnostic pass is made, acceptable runs are further analyzed. All data were taken in 20-minute runs using the so-called Wobble sourcetracking mode, which is optimal for observations of a pointlike source. In Wobble mode the source is positioned at a 0.5 offset from the center of the field of view of the camera during observations, which allows for both on-source observations and simultaneous estimation of the background contamination caused by charged CRs. The number of background events in the signal region was estimated using a number of regions distributed symmetrically with respect to the center of the camera for each wobble offset. The offset directions toward north, south, east, or west were consequently alternated on a run-by-run basis. A total of 74 data runs were collected at zenith angles between $26^{\circ}$ and $39^{\circ}$. The average zenith angles and the average event trigger rate were $31.5 \pm 3.7$ and $232 \pm 12 \mathrm{~Hz}$, respectively. Prior to applying analysis cuts, data were selected for adequate image quality, by requiring a minimum integrated charge of all pixels in the image of 400 digital counts (approximately 80 photo-electrons) and a maximum distance of the image's centroid from the center of the field of view of 1.43 . These cuts were a priori optimized using the Crab Nebula data sample. Each accepted event was also required to contain at least two images passing these cuts. The VERITAS experimental setup during Cassiopeia A observations included two telescopes placed at a rather small separation of $35 \mathrm{~m}$. Coincidence events including both of these telescopes have been removed from the analysis. During the summer of 2009 , one of these telescopes was relocated in order to improve the sensitivity of the array.

\section{DATA ANALYSIS}

The imaging analysis of the VERITAS data is based on the reconstruction of the shower direction for each individual event 
Table 1

Summary of Analysis Cuts

\begin{tabular}{lcccc}
\hline \hline Set & Flux $(\mathrm{Crab})$ & $\mathrm{MSW}\left({ }^{\circ}\right)$ & $\mathrm{MSL}\left({ }^{\circ}\right)$ & $\theta\left({ }^{\circ}\right)$ \\
\hline A & 0.03 & {$[0.05,1.08]$} & {$[0.05,1.19]$} & 0.13 \\
B & 1 & {$[0.05,1.1]$} & {$[0.05,1.39]$} & 0.158 \\
\hline
\end{tabular}

Table 2

Results of Data Analysis

\begin{tabular}{lcccc}
\hline \hline \multicolumn{1}{c}{ Source } & \multicolumn{2}{c}{ Crab Nebula } & \multicolumn{2}{c}{ Cas A } \\
\hline Exposure $(\mathrm{hr})$ & 3.0 & & 21.8 & $\mathrm{~B}$ \\
Set of cuts & $\mathrm{A}$ & $\mathrm{B}$ & $\mathrm{A}$ & 1277 \\
On events & 891 & 1298 & 625 & 6164 \\
Off events $^{\mathrm{a}}$ & 480 & 841 & 3538 & 7.0 \\
Significance $\sigma)$ & 47.6 & 50.5 & 8.3 & $0.191 \pm 0.028$ \\
$R_{\gamma}\left(\right.$ minute $\left.^{-1}\right)$ & $5.07 \pm 0.18$ & $7.10 \pm 0.22$ & $0.148 \pm 0.019$ & 0.77 \\
$R_{\mathrm{CR}}\left(\right.$ minute $\left.^{-1}\right)$ & 0.35 & 0.80 & 0.32 & \\
\hline
\end{tabular}

Note. ${ }^{\text {a }}$ A total number of Off events was accumulated over a few similar circular regions.

(Konopelko et al. 1999; Hofmann et al. 1999; Krawczynski et al. 2006), and joint parameterization of the shape of the Cherenkov light flash from an individual shower using a multiple-telescope approach (Konopelko 1995; Krawczynski et al. 2006). All recorded events were subjected to the canonical directional cut on $\theta^{2}$, where $\theta$ is the angular distance between the true source position on the sky and the reconstructed one. Of the remaining events, the candidates for $\gamma$-ray showers were selected using two simultaneously applied cuts on the parameters of image shape: mean-scaled width (MSW) and mean-scaled length (MSL). These three major analysis cuts were optimized using Crab Nebula observational data from the same epoch, chosen for the same zenith angle range as covered in observations of Cassiopeia A. The choice of optimal analysis cuts depends noticeably on the flux of the putative $\gamma$-ray source. Therefore, we developed two sets of analysis cuts, appropriate for flux levels of 1 and 0.03 Crab (see Table 1), respectively. Both sets of optimal analysis cuts yield comparable signal significances for the Crab Nebula as well as Cassiopeia A (Table 2).

The VERITAS array enables measurement of the arrival direction of every individual shower detected. All recorded events that have passed both the image quality cuts and specific analysis cuts can be plotted in a two-dimensional sky map. Even after applying rather strict selection criteria such maps are dominated by the flux of the isotropic CR background. A number of methods (background models) have been developed for effective removal of background (Berge et al. 2007). These models can effectively handle the background issues of diverse observations, but the weaknesses or strengths of any particular approach depend on the flux, angular morphology, and spectrum of a given $\gamma$-ray source. In this paper, we used a method that is rather stable with respect to any systematic background inhomogeneity across the camera field of view, the so-called ring-background model. In this model, a ring (annulus) around the location of a putative $\gamma$-ray source in the camera focal plane provides an immediate background estimate. The canonical angular radius of the background ring is 0.5 , whereas the angular area (solid angle) covered by the ring is typically chosen to be larger than that of the circular source region by a factor of 7-10. The excess map of the sky region around Cassiopeia A for the data set of $22 \mathrm{hr}$ is shown in Figure 1. An evident excess due to $\gamma$ rays at an $8.3 \sigma$ level of statistical significance (Li \& Ma 1983)

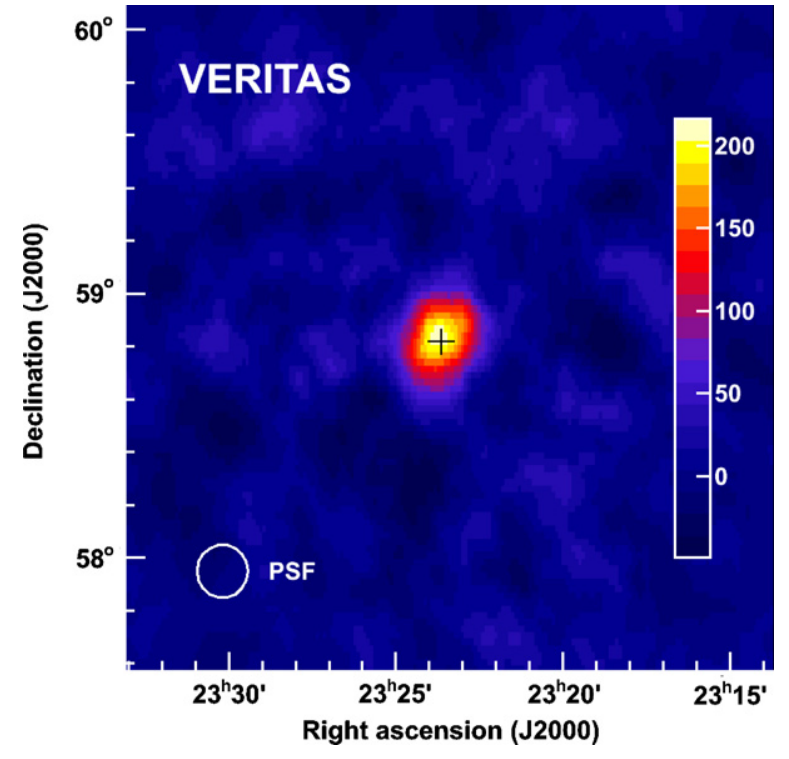

Figure 1. Smoothed sky map of excess counts from the region centered at Cassiopeia A observed with VERITAS for a total of $22 \mathrm{hr}$ in 2007. The color bar represents the excess event counts. The white circle indicates the size of the VERITAS point-spread function. The cross indicates the measured position of the $\mathrm{TeV} \gamma$-ray source. The radius of a smoothing circular window was 0.115 .

can be observed at the position of Cassiopeia A. This result has been cross-checked using a standard Wobble analysis.

\section{SOURCE LOCALIZATION}

The energy-averaged angular resolution of the VERITAS array for an individual $\gamma$-ray event is approximately $4^{\prime}-6^{\prime}(68 \%$ containment radius). This implies that a point-like $\gamma$-ray source detected by VERITAS appears as a spot of finite size in the expanded $\gamma$-ray sky map. The centroid of this spot is taken as the coordinates of the putative $\gamma$-ray emitter. Any error in the telescope pointing direction will deteriorate the exact measurement of the $\gamma$-ray source position. Note that the pointing accuracy of the telescopes is limited, by small misalignment of azimuth and altitude axes, and elastic deformations of the telescope structure. These effects contribute to the mispointing of the array, which strongly depends on the altitude and azimuth of observation. Most of the pointing uncertainties can be substantially diminished by taking pointing calibration data on a monthly basis. Each telescope is pointed at a number of bright stars uniformly distributed on the sky. The star is imaged by the telescope mirror onto a screen in front of the Cherenkov camera, and the image is recorded by a CCD camera. The position of each spot is then compared to the nominal center of the Cherenkov camera. These results contribute to a multi-parameter pointing model in the telescope tracking software that corrects for the measured misalignment during observation. This procedure was extensively tested on a number of VHE $\gamma$-ray point sources of known position. In addition, the residual mispointing can be evaluated from a detailed comparison of the nominal position of the source evaluated for the different wobble offsets, energy thresholds, telescope multiplicities, analysis cuts, etc. During Cassiopeia A observations the systematic pointing error of the VERITAS array is $\sim 1$ '.2.

The measured position of the $\gamma$-ray source is determined by a fit over a circular window of 0.5 radius centered on Cassiopeia A using its known coordinates. The profile of the $\gamma$-ray excess 
can be modeled by the two-dimensional Gaussian distribution:

$$
f\left(\theta_{x}, \theta_{y}\right) \propto \exp \left(-\frac{1}{2}\left(\frac{\left(\theta_{x}-\theta_{x o}\right)^{2}}{\sigma_{x}^{2}}+\frac{\left(\theta_{y}-\theta_{y_{o}}\right)^{2}}{\sigma_{y}^{2}}\right)\right),
$$

where $\theta_{x_{o}}, \theta_{y_{o}}$ are the angular coordinates of the $\gamma$-ray emission centroid, and $\sigma_{x}^{2}, \sigma_{y}^{2}$ are the extensions of the signal region in two perpendicular directions. The width of the two-dimensional Gaussian fit is composed of the fixed angular resolution of the VERITAS array and the intrinsic size of the source. Note that the excess map generated by the ring-background model has been smoothed using a circular window of 0.115 radius, which approximately corresponds to the angular resolution of the VERITAS array. First, this method was tested on the 1ES 2344+514 data taken with the VERITAS telescope array during the same observational season as that of Cassiopeia A with an instrument of similar configuration. 1ES $2344+514$ is a blazar-type active galactic nucleus, which was in a high state of $\gamma$-ray emission during the Cassiopeia A observations. Given the redshift of 1 ES $2344+514$ of $z=0.044$, it is indubitably a point source. In addition, the total number of recorded $\gamma$ rays from 1ES 2344+514 was of the same order as the number of excess counts from Cassiopeia A. Therefore, this object could be used as a calibration source for estimating the limits of the source localization procedure. The width of the $\gamma$-ray PSF evaluated using 1ES $2344+514$ data is $\sigma_{o}=4.8$. However, this observationally determined PSF is significantly affected by the angular size of the signal region used by the ringbackground model, which was adopted for the smoothing of the two-dimensional sky maps. Alternatively, one can use the excess count sky map of uncorrelated bins which leads to similar results. The position of the 1ES $2344+514 \gamma$-ray peak derived from the best fit was found to be consistent with the astronomical position of this object (R.A. $(\alpha)$ : $23^{\mathrm{h}} 47^{\mathrm{m}} 04^{\mathrm{s}} .919$, decl. $\left.(\delta):+51^{\circ} 42^{\prime} 17^{\prime \prime} .87\right)$ within the statistical uncertainties of the best-fit position on right ascension and declination, $\Delta_{\text {R.A. }}$. and $\Delta_{\text {dec }}$, of 1 '.24 and $9^{\prime \prime}$, respectively. These results ultimately validate the accuracy of the analysis method. Cassiopeia A data were analyzed using exactly the same two-dimensional analysis technique. The derived position of the peak of $\gamma$-ray emission from Cassiopeia A deviates from the nominal position of the SNR (Becker et al. 1991; $\left(\alpha=23^{\mathrm{h}} 23^{\mathrm{m}} 24^{\mathrm{s}}, \delta=+58^{\circ} 48^{\prime} .9\right)$ by less than $\Delta_{\text {R.A. }}=14^{\prime \prime}$ and $\Delta_{\text {dec }}=35^{\prime \prime}$. Evidently, the observed $\gamma$-ray emission is associated with the Cassiopeia A SNR.

\section{SOURCE EXTENSION}

The angular radius of Cassiopeia $\mathrm{A}$, measured at wavelengths longer than those corresponding to TeV energies, is about 2'.5. Primarily, this can be used as a characteristic angular size of the $\mathrm{TeV} \gamma$-ray source. It is apparent that such a small angular dimension of Cassiopeia $\mathrm{A}$ is well below the angular resolution (PSF) of VERITAS, $\sigma_{\circ} \simeq 4.8$, which unavoidably smears out the intrinsic source distribution and consequently does not permit detailed mapping of the morphology of the $\gamma$-ray source. The angular profile of the observed $\gamma$-ray peak finally constrains the intrinsic angular size of the source. The two-dimensional, azimuthally symmetric Gaussian function could be naturally used to model the measured angular shape of the $\gamma$-ray signal. The angular extent of the $\gamma$-ray peak toward Cassiopeia A measured with VERITAS is $\sigma_{\text {Cas A }}=5.3 \pm 0.5$.

The angular extent of the PSF can be derived from the data taken on a calibration $\gamma$-ray source. For that we can use again the contemporaneous observations of 1ES 2344+514. The best fit of the $\gamma$-ray peak for $1 E S 2344+514$ gives $\sigma=4.8 \pm 0$.'2. If we assume a $\gamma$-ray source with a Gaussian profile, an approximate upper limit on the source extent can be calculated by summing the measured extents of the PSF and the Cassiopeia A $\gamma$-ray signal in quadrature, $\sigma_{s}=\left(\sigma_{\text {Cas A }}^{2}-\sigma^{2}\right)^{1 / 2} \lesssim 3$ '.5. Thus, given rather large statistical errors of the involved angular extents the shape of the Cassiopeia A signal is hardly distinguishable from the PSF and the $\gamma$-ray signal is statistically consistent with the point source.

The low statistics of currently recorded $\gamma$ rays from Cassiopeia A are not sufficient to draw a final conclusion on the source extension. A further, deeper, observation of Cassiopeia A with VERITAS might help to improve the measurement of the angular extension of the TeV $\gamma$-ray source.

\section{ENERGY RECONSTRUCTION}

Stereoscopic observations of atmospheric showers with four VERITAS telescopes enable accurate localization of the shower axis in the ground plane. Thus, the impact distances from the shower axis to the system telescopes can be calculated in a straightforward manner. The generic reconstruction algorithm (Konopelko et al. 1999) is based on a simultaneous use of image orientation in several telescopes for each individual event. The accuracy of such reconstruction is limited by the uncertainties in the determination of the image orientation. By observing $\gamma$-ray showers at zenith angles less than $45^{\circ}$ and restricting the impact distances to less than $250 \mathrm{~m}$, the average accuracy in evaluation of the telescope impacts is better than $10 \mathrm{~m}$. If the distance from the shower axis to the telescope $\left(r_{i}, i=1, n\right.$, where $n$ is the number of recorded images) is known, the primary energy of the air shower can be evaluated using the inverse function of the image size with respect to the shower energy $E_{i}=F\left(S_{i}, r_{i}, \theta\right)$ (Konopelko et al. 1999). Here, $S_{i}$ stands for the image size (the total number of photoelectrons in the image), $r_{i}$ is the impact distance, and $\theta$ is the zenith angle. This function can be well represented by a multi-variable lookup table, which contains the mean energy for Monte Carlo simulations across the range of image sizes and impact distances of recorded $\gamma$-ray showers. Such lookup tables were created for a number of zenith angles. Finally, the shower energy can be computed by averaging over all reconstructed energies for individual telescopes $E_{i}, i=$ $1, n$, as $E_{o}=\sum_{i} w_{i} E_{i}$, where $w_{i}$ is the statistical weight $\left(\sum_{i} w_{i}=1\right)$. Rather accurate and robust estimations can be achieved for $w_{i}=1 / n$. The energy resolution of the VERITAS array of four imaging air Cherenkov telescopes averaged over the entire dynamic energy range is $15 \%-20 \%$. Note that the energy resolution is unavoidably limited by the fluctuations in image size for a given shower energy.

In order to control any possible systematic biases in the energy reconstruction, one can use the error in the reconstructed energy, $\delta E=\left(E-E_{o}\right) / E_{o}$ as a function of the true energy, $E_{o}$. Even though this error usually does not exceed a $5 \%$ level over the energy range from $150 \mathrm{GeV}$ to $10 \mathrm{TeV}$, a positive bias can be observed at energies close to the threshold and a negative dropoff can be seen at very high energies (VHEs). These biases are an intrinsic feature of the reconstruction algorithms and have been well understood using detailed Monte Carlo simulations. To diminish any noticeable effect of these biases on the measured energy spectrum, one can limit the lowest and highest energy by requiring that the energy bias does not exceed $20 \%$ (see, e.g., Aharonian et al. 2006). Note that the effective energy range chosen for the spectrum evaluation substantially depends on the 
zenith angle of observations as well as on the actual setup of a system configuration.

\section{SPECTRAL ANALYSIS}

Despite the fact that stereoscopic observations with four VERITAS telescopes provide very efficient rejection of the CR background, the sample of selected $\gamma$-ray-like events still contains a substantial fraction of background CRs. In order to remove any effect of background on the reconstructed energy spectrum of $\gamma$ rays, a similar energy reconstruction procedure has to be applied to the events acquired from a number of purely background regions as defined in the wobble-mode analysis. This enables a proper estimate of the background contamination, which has to be subtracted from the signal region. The resulting energy-dependent $\gamma$-ray rate can be used for the spectrum evaluation by applying a specific response matrix, which handles various zenith angles, system configurations, observational modes, analysis setups, etc. Such a response matrix represents a complete set of effective collection areas of the instrument, which can be derived using detailed Monte Carlo simulations. The CORSIKA shower simulation code (see Maier et al. 2007) was used to generate the $\gamma$-ray-and CR-induced air showers over the accessible range of zenith angles and in the energy range between $50 \mathrm{GeV}$ and $100 \mathrm{TeV}$, assuming the $\gamma$-ray energy spectrum to be a power law with an index of 2.0. Simulations of the VERITAS response were carried out using the GrISU code, developed by the Grinnell College and Iowa State University groups (e.g., see Maier et al. 2007). Simulations were compared with data in great detail. In order to avoid any remaining small energy biases in the energy reconstruction discussed above, it was necessary to compute the effective collection areas as a function of the reconstructed energy rather than true shower energy (see, e.g., Aharonian et al. 2006). This helps to complete the unfolding of the intrinsic source spectrum.

The complete spectrum evaluation procedure has been tested on the Crab Nebula data taken during 2007/2008 observation season. The result is consistent with the previous measurements of the Crab Nebula spectrum with HEGRA (Aharonian et al. 2004), HESS (Aharonian et al. 2006), and MAGIC (Albert et al. 2008), as well as measurements made using VERITAS data but different analysis tools. Spectral analysis of the Cassiopeia A data leads to the energy spectrum

$$
\begin{aligned}
& \frac{d N_{\gamma}}{d E}=(1.26 \pm 0.18) \times 10^{-12}(E / 1 \mathrm{TeV})^{-2.61 \pm 0.24_{\text {stat }} \pm 0.2_{\text {sys }}} \\
& \mathrm{cm}^{-2} \mathrm{~s}^{-1} \mathrm{TeV}^{-1}
\end{aligned}
$$

with the flux normalization

$$
F_{\gamma}(>1 \mathrm{TeV})=(7.76 \pm 0.11) \times 10^{-13} \mathrm{~cm}^{-2} \mathrm{~s}^{-1}
$$

A power-law fit to the Cassiopeia A spectrum yields a $\chi^{2}=$ 2.15 for 4 degrees of freedom with a chance probability of $P=0.71 \%$. This result is in good agreement with the HEGRA spectrum estimate (Aharonian et al. 2001) as well as the recently published MAGIC spectrum (Albert et al. 2007; see Figure 2). Currently, the rather limited exposure on Cassiopeia A with VERITAS limits spectral measurements to the energy range from $300 \mathrm{GeV}$ to $5 \mathrm{TeV}$. The spectrum measured over this interval does not reveal any break of a spectral slope or cutoff. Limiting the upper energy bound for the spectral fit at $3 \mathrm{TeV}$ results in a flatter spectrum index $(\Gamma=2.4)$, even though the combination of the statistical and systematic errors does not

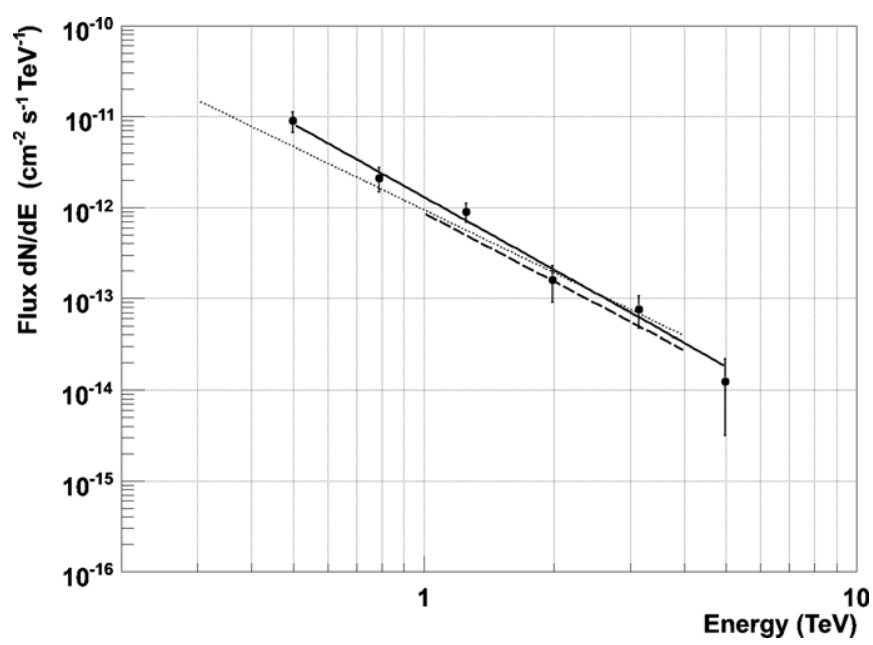

Figure 2. Differential energy spectrum of $\mathrm{TeV} \gamma$ rays from Cassiopeia A, measured with VERITAS. Also shown are the HEGRA (dashed line) and the MAGIC (dotted line) energy spectra adapted from Aharonian et al. (2001) and Albert et al. (2007), respectively.

allow us to draw any firm conclusion. Despite the fact that no $\gamma$-ray events were detected above $6 \mathrm{TeV}$, the statistical significance of this result is not sufficient for any firm statement regarding possible deviation of the spectral shape from a simple power law. Any other, more sophisticated, multi-parameter fit functions are not favored over a simple power law. Adding to the spectral fit an exponential cutoff term, $d N_{\gamma} / d E \propto E^{-\Gamma} e^{-E / E_{o}}$, does not improve the result but rather degrades the quality of the fit. For the cutoff energy $E_{o}=2 \mathrm{TeV}(\Gamma=2.35)$ the fit gives the $\chi^{2}$-value of $3.1(P=0.54 \%)$. Further reduction of the cutoff energy down to $E_{o}=1 \mathrm{TeV}(\Gamma=1.46)$ destroys the spectral fit $\left(\chi^{2}=5.4, P=0.25 \%\right)$. This result shows that the value of possible high-energy cutoff in the Cassiopeia A spectrum is outside the energy range measured here. Future observations with VERITAS will help to extend the spectral measurements for Cassiopeia A SNR.

\section{DISCUSSION}

Understanding the mechanism of particle acceleration in isolated SNR shocks is of great interest. The question of whether or not the VHE $\gamma$-ray emission of Galactic SNRs implies a sufficiently high flux of charged CRs, merging into a steady flux of Galactic CRs, remains one of the most stimulating rationales for ground-based $\gamma$-ray astronomy. Following initial, simplified estimates of the expected $\gamma$-ray flux from Galactic SNR (Drury et al. 1994), more refined models have been developed to describe particle acceleration in, and high-energy emission from, Cassiopeia A (e.g., Atoyan et al. 2000a; Berezhko et al. 2003). Cassiopeia A is one of the best-studied SNR in the Galaxy, and a multitude of observations in different wavebands constrain its physical properties and hence the environment in which particle-acceleration processes operate. The analysis of scattered optical light indicates that Cassiopeia A was of Type IIb and originated from the collapse of the helium core of a red supergiant that had lost most of its hydrogen envelope before exploding (Krause et al. 2008).

Cassiopeia A is a very bright radio (Bell et al. 1975) and X-ray source (Holt et al. 1994). Borkowski et al. (1996) have modeled the thermal X-ray emission, including the present size and expansion rate. They concluded that the fast wind of the final blue-supergiant stage of the progenitor has swept 
into a dense shell the wind material from the earlier redsupergiant phase. The SNR blast wave has already passed through and accelerated the dense $\left(n_{H} \simeq 15 \mathrm{~cm}^{-3}\right)$ shell of circumstellar material (CSM). About $8 M_{\odot}$ of X-ray-emitting swept-up gas is found in Cassiopeia A, mostly in the form of heavy elements and predominantly located in the CSM shell and the outer, unperturbed, red-supergiant wind (Willingale et al. 2003). The presence of a jet, numerous slow-moving flocculi, and the general asymmetry of the remnant requires careful threedimensional modeling of the SN explosion (Laming \& Hwang 2003; Young et al. 2006), which has recently been used to demonstrate the absence of a Wolf-Rayet (WR) phase of the progenitor van Veelen et al. (2009).

The high-gas density combined with the high-radio flux observed from Cassiopeia A permit an estimate of the magnetic field strength, because the radio-emitting electrons must produce non-thermal bremsstrahlung between $100 \mathrm{MeV}$ and $10 \mathrm{GeV}$ (Cowsik \& Sarkar 1980). An upper limit on the GeV-band flux has been derived using EGRET data (Esposito et al. 1996), which in a one-zone model leads to a lower limit on the magnetic field, $B \gtrsim 0.4 \mathrm{mG}$ (Atoyan et al. 2000a). A high-magnetic-field strength strongly limits the flux of an inverse-Compton emission component in the $\mathrm{TeV}$ band on account of its direct relation to synchrotron X-ray emission (Pohl 1996), which is observed up to $120 \mathrm{keV}$ (Allen et al. 1997; Favata et al. 1997). The observed hard X-ray emission also includes lines from the ${ }^{44} \mathrm{Ti}$ decay chain (Renaud et al. 2006).

The non-thermal X-ray emission predominantly originates from filaments and knots in the reverse-shock region of Cassiopeia A (Helder \& Vink 2008), some of which are variable in flux on timescales of years (Uchiyama \& Aharonian 2008). Both the filaments themselves and their flux variability require a strong magnetic field, but estimates of its exact amplitude depend on their detailed interpretation. Typically, one obtains somewhat higher values for the magnetic-field strength, if one assumes the size and variability timescale are determined by electron energy losses (Vink \& Laming 2003; Uchiyama et al. 2007; Uchiyama \& Aharonian 2008), as opposed to scenarios involving the rapid damping of a turbulently amplified field (Pohl et al. 2005) or localized spikes in dynamical magnetic turbulence (Bykov et al. 2008). The complicated structure of Cassiopeia A, including fast-moving clumps of ejecta and knots of high radio brightness, has triggered the development of many scenarios involving first- and second-order Fermi-type acceleration at various locations (e.g., Scott \& Chevalier 1975; Jones et al. 1994; Atoyan et al. 2000b).

The presence of a large flux of high-energy electrons in the reverse-shock region, responsible for the non-thermal radio-to$\mathrm{X}$-ray emission, will also produce high-energy $\gamma$-ray emission through non-thermal bremsstrahlung and inverse-Compton scattering (e.g., Atoyan et al. 2000a). Based on that leptonic emission, Cassiopeia A would appear in VERITAS data as a diskor ring-like source with outer radius $R_{l} \lesssim 2^{\prime}$ (Uchiyama \& Aharonian 2008). If, on the other hand, the VHE $\gamma$-ray emission from Cassiopeia A were dominated by $\pi^{\circ}$-decay photons produced in inelastic collisions of relativistic protons, the location of the particle-acceleration sites is less constrained by data in other wavebands, and substantial acceleration of CR protons could proceed at the outer blast wave (Berezhko et al. 2003). The size of Cassiopeia A in VERITAS data could therefore be slightly larger than for leptonic scenarios, with an outer radius $R_{h} \lesssim 2.5$. However, both predicted angular extensions are substantially less than the current angular resolution of VERITAS.
The VERITAS data are consistent with a point-like $\gamma$-ray source. Since the extended $\mathrm{TeV} \gamma$-ray emission has not been resolved with VERITAS yet, current results remain fully consistent with the hypothesis that the VHE $\gamma$-ray emission originates from the central part of Cassiopeia A, where a compact object has been observed at longer wavelengths. Nevertheless, we encourage modeling the spatial distribution of VHE $\gamma$-ray emission from Cassiopeia A in preparation for the next generation of imaging atmospheric Cherenkov telescopes, $\mathrm{CTA}^{29}$ and AGIS, ${ }^{30}$ both of which are projected to have an angular resolution better than that of VERITAS by a factor of at least 2 .

None of the published calculations of VHE $\gamma$-ray production correctly predict both the flux and the spectrum observed with VERITAS. Atoyan et al. (2000a, 2000b) have carefully modeled the acceleration, propagation, and photon-emission spectra of high-energy electrons. For the parameters chosen for their displayed $\gamma$-ray spectra, the TeV-band emission is a mixture of non-thermal bremsstrahlung and inverse-Compton scattering that would account for about $25 \%$ of the flux observed with VERITAS and feature a softer spectrum than observed $\left(\propto E_{\gamma}^{-3.2}\right)$. We can speculate that a better fit may be achieved by a small reduction of the magnetic-field strength and/or an increase in the cutoff energy of the electron injection spectrum, which astrophysically is determined by the details of the acceleration process, the magnetic-field strength in the acceleration region, and efficiency limitations imposed by the geometry of the acceleration region. However, a low GeV-band flux measurement or upper limit derived with Fermi-LAT would imply a magnetic field stronger than that assumed by Atoyan et al. (2000a).

Models of hadronic VHE $\gamma$-ray emission are somewhat less constrained by radio and X-ray data than are their leptonic counterparts. An indirect relation exists in that a very efficient acceleration of CR nuclei by shock fronts leads to a modification of such shocks, resulting in relatively soft spectra below a GeV particle energy and rather hard spectra around a $\mathrm{TeV}$ (e.g., Blandford \& Eichler 1987; Berezhko \& Ellison 1999), although a CR-induced strong magnetic field can substantially reduce the shock modification compared with the naive unmagnetized case (Caprioli et al. 2008). The soft radio spectrum observed from Cassiopeia A is indeed consistent with nonlinear kinetic models of CR acceleration in SNR (e.g., Berezhko et al. 2003). However, the hard spectra predicted beyond a particle energy of $1 \mathrm{TeV}$ have not been observed to date. In fact, the VHE $\gamma$-ray spectra measured from shell-type SNR are all well described by either a power law with photon index $s \lesssim-2.2$, or a power law with gradual roll-off (e.g., Huang et al. 2007); this suggests that the acceleration of CR protons beyond $1 \mathrm{TeV}$ must be less efficient than previously thought, if the observed VHE $\gamma$-ray emission completely arises from interactions of these protons.

Except for the electron energy losses, the high-energy cutoff in the spectrum of accelerated protons is determined by the same physical processes as that of the electrons, the details of which are not well understood. The published models may therefore be too optimistic in predicting the cutoff energy (e.g., Berezhko et al. 2003). In any case, more work is required to better understand the high-energy end of CR proton spectra accelerated in SNR (e.g., Ellison \& Vladimirov 2008). In addition, the role stochastic particle acceleration plays in SNR needs to be explored in more detail (Liu et al. 2008). Observationally, the

\footnotetext{
29 http://www.cta-observatory.org

30 http://www.agis-observatory.org
} 
next step toward a better understanding of particle acceleration in Cassiopeia A will be measuring the GeV-band $\gamma$-ray spectrum with the Fermi-LAT.

This research was supported by grants from the U.S. Department of Energy, the U.S. National Science Foundation, and the Smithsonian Institution, by NSERC in Canada, by Science Foundation Ireland, and by STFC in the UK. The VERITAS collaboration acknowledges the NASA support on the Fermi GST LAT grant No. NNX08AV62G.

\section{REFERENCES}

Aharonian, F., et al. 2001, A\&A, 370, 112

Aharonian, F., et al. 2004, ApJ, 614, 897

Aharonian, F., et al. 2006, A\&A, 457, 899

Albert, J., et al. 2007, A\&A, 474, 937

Albert, J., et al. 2008, ApJ, 674, 1037

Allen, G. E., et al. 1997, ApJ, 487, L97

Ashworth, W. B. 1980, J. Hist. Astrn., 11, 1

Atoyan, A. M., Aharonian, F. A., Tuffs, R. J., \& Völk, H. J. 2000a, A\&A, 355, 211

Atoyan, A. M., Tuffs, R. J., Aharonian, F. A., \& Völk, H. J. 2000b, A\&A, 354 915

Becker, R. H., White, R. L., \& Edwards, A. L. 1991, ApJS, 75, 1

Bell, A. R., Gull, S. F., \& Kenderdine, S. 1975, Nature, 257, 463

Berezhko, E. G., \& Ellison, D. C. 1999, ApJ, 526, 385

Berezhko, E. G, Pühlhofer, G., \& Völk, H. J. 2003, A\&A, 400, 971

Berge, D., Funk, S., \& Hinton, J. 2007, A\&A, 466, 1219

Blandford, R. D., \& Eichler, D. 1987, Phys. Rep., 154, 1

Borkowski, K. J., Szymkowiak, A. E., Blondin, J. M., \& Sarazin, C. L. 1996, ApJ, 466, 866

Bykov, A. M., Uvarov, Y. A., \& Ellison, D. C. 2008, ApJ, 689, L133

Caprioli, D., Blasi, P., Amato, E., \& Vietri, M. 2008, ApJ, 679, L139

Cowsik, R., \& Sarkar, S. 1980, MNRAS, 191, 855

Davies, J. M., \& Cotton, E. S. 1957, J. Sol. Energy, 1, 16

Drury, L. O., Aharonian, F. A., \& Völk, H. J. 1994, A\&A, 287, 959

Ellison, D. C., \& Vladimirov, A. 2008, ApJ, 673, L47

Esposito, J. A., et al. 1996, ApJ, 461, 820

Favata, F., et al. 1997, A\&A, 324, L49

Fesen, R. A., et al. 2006, ApJ, 645, 283

Goret, P., Gouiffes, C., Nuss, E., \& Ellison, D. C. 1999, Proc. 26th ICRC (Salt Lake City), 3, 496

Helder, E. A., \& Vink, J. 2008, ApJ, 686, 1094
Hofmann, W., et al. 1999, Astropart. Phys., 12, 135

Holder, J. et al. (VERITAS Collaboration) 2006, Astropart. Phys., 25, 361

Holt, S. S., Gotthelf, E. V., Tsunemi, H., \& Negoro, H. 1994, PASJ, 46, L151

Huang, C.-Y., Park, S.-E., Pohl, M., \& Daniels, C. D. 2007, Astropart. Phys., 27,429

Hughes, J. P., Rakowski, C. E., Burrows, D. N., \& Slane, P. O. 2000, ApJ, 528, L109

Jones, T. W., Kang, H., \& Tregillis, I. L. 1994, ApJ, 432, 194

Kassim, N. E., Perley, R. A., Dwarakanath, K. S., \& Erickson, W. C. 1995, ApJ, 455, L59

Konopelko, A. 1995, in Proc. Padova Workshop on TeV Gamma-Ray Astrophysics, Towards a Major Atmospherics Cherenkov Detector-IV, Padova, Italy, ed. M. Cresti, 373

Konopelko, A., et al. 1999, Astropart. Phys., 10, 275

Krause, O., et al. 2008, Science, 320, 1195

Krawczynski, H., et al. 2006, Astropart. Phys., 25, 380

Laming, J. M., \& Hwang, U. 2003, ApJ, 597, 347

Lessard, R. W., et al. 1999, Proc. 26th ICRC (Salt Lake City), 3, 488

Li, T.-P., \& Ma, Y.-Q. 1983, ApJ, 272, L317

Liu, S., Fan, Z.-H., Fryer, C. L., Wang, J.-M., \& Li, H. 2008, ApJ, 683, L163

Maier, G., et al. 2007, in Proc. 30th ICRC (Merida), 1413

McCann, A., Hanna, D., Kildea, J., \& McCutcheon, M. 2009, Astropart. Phys., 32,325

Mezger, P. G., Tuffs, R. J., Chini, R., Kreysa, E., \& Gemuend, H.-P. 1986, A\&A, 167,145

Pohl, M. 1996, A\&A, 307, L57

Pohl, M., Yan, H., \& Lazarian, A. 2005, ApJ, 626, L101

Reed, J. E., Hester, J. J., Fabian, A. C., \& Winkler, P. F. 1995, ApJ, 440, 706

Renaud, M., et al. 2006, ApJ, 647, L41

Reynolds, P. T., et al. 1993, ApJ, 404, 206

Scott, J. S., \& Chevalier, R. 1975, ApJ, 197, L5

Stephenson, F. R., \& Green, D. A. 2002, The Historical Supernova Remnants (Cambridge: Cambridge Univ. Press)

Tuffs, R. J., Drury, L. O. C., Fischera, J., Heinrichsen, I., Russel, S., \& Völk, H. J. 1997, in Proc. 1st ISO Workshop on Analytical Spectroscopy, ed. A. M Heras, K. Leech, N. R. Trams, \& M. P. Noordwijk (ESA SP-419; Noordwijk: ESA), 177

Uchiyama, Y., \& Aharonian, F. A. 2008, ApJ, 677, L105

Uchiyama, Y., et al. 2007, Nature, 449, 576

van Veelen, B., Langer, N., Vink, J., García-Segura, G., \& van Marle, A. J. 2009, A\&A, 503, 495

Vink, J., \& Laming, J. M. 2003, ApJ, 584, 758

Vink, J., et al. 2001, ApJ, 560, L79

Weekes, T. et al. (VERITAS Collaboration) 2002, Astropart. Phys., 17, 221

Willingale, R., Bleeker, J. A. M., van der Heyden, K. J., \& Kaastra, J. S. 2003, A\&A, 398, 1021

Young, P. A., Fryer, C. L., \& Hungerford, A. 2006, ApJ, 640, 891 